\title{
1 Single-cell RNA-seq reveals early heterogeneity during ageing
}

\section{2 in yeast}

3

5

6

7

8

Yi Zhang ${ }^{1 \#}$, Jincheng Wang ${ }^{1 \#}$, Yuchen Sang ${ }^{1}$, Shengxian Jin $^{1}$, Xuezheng Wang ${ }^{2,3}$, Gajendra Kumar Azad ${ }^{4,5}$, Mark A. McCormick ${ }^{6,7}$, Brian K. Kennedy ${ }^{4,8,9}$, Qing Li ${ }^{2}$, Jianbin Wang $^{10}$, Xiannian Zhang ${ }^{11 *}$, and Yanyi Huang ${ }^{1,12-14 *}$

1 Beijing Advanced Innovation Center for Genomics (ICG), Biomedical Pioneering Innovation Center (BIOPIC), Peking-Tsinghua Center for Life Sciences, School of Life Sciences, Peking University, Beijing 100871, China

2 State Key Laboratory of Protein and Plant Gene Research, School of Life Sciences and Peking-Tsinghua Center for Life Sciences, Peking University, Beijing, China 100871

3 Academy for Advanced Interdisciplinary Studies, Peking University, Beijing, China 100871

4 Departments of Biochemistry, Yong Loo Lin School of Medicine, National University of Singapore, Singapore, 117596

5 Department of Zoology, Patna University, Patna, Bihar, India, 800005

6 Department of Biochemistry and Molecular Biology, School of Medicine, University of New Mexico Health Sciences Center, Albuquerque, NM, 87131, USA

7 Autophagy Inflammation and Metabolism Center of Biomedical Research Excellence, Albuquerque, NM 87131, USA.

8 Healthy Longevity Programme, Yong Loo Lin School of Medicine, National University of Singapore 
9 Centre for Healthy Longevity, National University Health System, Singapore, 119228

33

10 School of Life Sciences, Beijing Advanced Innovation Center for Structural Biology,

Tsinghua University, Beijing 100084, China

11 School of Basic Medical Sciences, Beijing Advanced Innovation Center for Human Brain

12 Analytical Chemistry, College of Chemistry, Peking University, Beijing 100871, China

\#These authors contributed equally to this work. 


\section{Abstract}

The budding yeast Saccharomyces cerevisiae has relatively short lifespan and is genetically tractable, making it a widely used model organism in ageing research. Here, we carried out a systematic and quantitative investigation of yeast ageing with single-cell resolution through transcriptomic sequencing. We optimized a single-cell RNA sequencing (scRNA-seq) protocol to quantitatively study the whole transcriptome profiles of single yeast cells at different ages, finding increased cell-to-cell transcriptional variability during ageing. The single-cell transcriptome analysis also highlighted key biological processes or cellular components, including oxidation-reduction process, oxidative stress response (OSR), translation, ribosome biogenesis and mitochondrion that underlie ageing in yeast. Remarkably, we uncovered a molecular marker, FIT3, that was linked to mitochondrial DNA loss and indicated the early heterogeneity during ageing in yeast. We also analyzed the regulation of transcription factors and further characterized the distinctive temporal regulation of the OSR by YAP1 and proteasome activity by RPN4 during ageing in yeast. Overall, our data profoundly reveal early heterogeneity during ageing in yeast and shed light on the ageing dynamics at the single cell level.

\section{Introduction}

It has been known for a long time that budding yeast Saccharomyces cerevisiae have limited division potential, only producing a finite number of daughter cells before death 1 . This phenomenon is defined as replicative ageing, and the number of daughter cells produced before death is defined as the replicative lifespan (RLS) ${ }^{2}$. Owing to its relatively short lifespan, detailed knowledge of its biology and its easy genetic manipulation, $S$. cerevisiae is regarded as an ideal model organism to study ageing ${ }^{3}$. Indeed, many ageing genes and signaling pathways initially found in yeast have also been later found to be conserved in other organisms, such as C. elegans, M. musculus and even humans ${ }^{4}$.

A dilemma of replicative ageing research in yeast exists between the rarity of old cells among an exponentially growing population either on a solid agar plate or in liquid media and the large number of pure old cells conventionally required for biochemical, genomic or transcriptomic analysis. To address this problem, several approaches have been developed to enrich old yeast cells, including magnetic sorting, elutriation, genetic programming and even computation ${ }^{5-9}$. However, these methods have yet to be successful at simultaneously ensuring both the quantity and purity of the isolated old yeast cells much less distinguishing old but living cells from dead ones. In addition, conventional bulk population analysis of ageing yeast cells may likely obscure some specific features within sub-populations due to the average effect ${ }^{10}$. Recent advances in microfluidics and single- 
cell imaging have revealed some phenotypic details of replicative ageing in yeast ${ }^{11-14}$; however, a systematic and quantitative investigation of yeast ageing at the single-cell and transcriptome level would be highly valuable.

Here, we developed a single-cell RNA-seq approach to study the replicative ageing of yeast and quantitatively assessed the heterogeneity between single yeast cells. Instead of partially purifying millions of old cells, exploiting single-cell technologies enabled us to obtain novel insights into yeast ageing from hundreds of single cells with precise ages. By profiling the transcriptomic landscapes of single yeast cells, we observed an increased cell-to-cell transcriptional variability and identified key functional biological processes or cellular components that were highly enriched during ageing. We also found early heterogeneity during ageing, indicated by a molecular marker of iron transport linked to mitochondrial DNA loss, and successfully characterized the distinctive temporal regulation of transcription between slow-dividing and fast-dividing age subgroups.

\section{Results}

Isolation of single yeast cells during ageing and scRNA-seq. Single yeast cells from isogenic populations ultimately have different lifespans. In fact, this is a universal phenomenon of ageing across species, albeit in different forms and ranges. And previous single-cell imaging data of replicative ageing in yeast have provided evidence of such heterogeneity. For example, when re-analyzing the single cell imaging data from the microfluidic-based yeast ageing studies ${ }^{11,12}$, we can observe that as early as $8 \mathrm{hr}$ after birth, the distribution of generations of single yeast cells had already become dispersed, and the ranges of the distribution gradually increased at $12 \mathrm{hr}$ and $16 \mathrm{hr}$ after birth (Supplementary Fig. 1a), showing that some cells always divided more rapidly than others ever since early in life. These early-stage cell division dynamics in yeast seems closely associated with replicative age, with a positive correlation between the generations at early time points (8hr, $12 \mathrm{hr}, 16 \mathrm{hr})$ after birth and the RLS $\left(\mathrm{R}=0.46,0.64,0.73 ; \mathrm{P}=9.6 \times 10^{-5}, 7.7 \times 10^{-9}, 7.7 \times 10^{-9}\right.$; Supplementary Fig. $1 \mathrm{~b}$ ) at the single-cell level. This new finding is consistent with the previous report that the division time of single yeast cells early in life is negatively correlated with RLS, and the division time increases dramatically when approaching the end of life ${ }^{11}$. It was also reported previously that early in life, the gene expression level of HSP104, which encodes a molecular chaperone that maintains proteostasis in yeast, negatively correlates with RLS ${ }^{11,12}$. Accordingly, after re-analyzing the single cell imaging data ${ }^{11,12}$, we also observed a negative correlation between the generations at early time points during ageing and the HSP104 gene expression level indicated by a GFP tag fused to this gene in single yeast cells $\left(R=-0.43,-0.51,-0.56 ; P=2.8 \times 10^{-4}, 8.4 \times 10^{-6}, 7.8 \times 10^{-7}\right.$; Supplementary Fig. 1c). Collectively, these single-cell imaging data indicate an early heterogeneity of cell 
divisions during ageing in yeast, and that the division dynamics early in life can predict lifespan.

To probe more deeply into the mechanisms underlying this early heterogeneity revealed by single-cell imaging, we further developed and applied scRNA-seq for transcriptome profiling of yeast during ageing (Fig. 1a; see Methods). We first conducted an RLS assay by continually performed manual microdissection of single yeasts on a solid agar plate ${ }^{15}$. At three different time points ( $2 \mathrm{hr}, 16 \mathrm{hr}$ and $36 \mathrm{hr}$ after birth), we manually isolated single ageing yeast cells from the plate and placed the cells individually into a single tube prefilled with lysis buffer containing an external RNA control consortium (ERCC) spike-in for assessing technical noise then followed the Smart-seq2-based protocol ${ }^{16,17}$ with refined modifications and optimization for yeast ageing research (see Methods).

In total, we collected 136 yeast ageing cells for sequencing. The timepoints of isolation and number of generations at that time were precisely recorded for each cell (Supplementary Table. 1). After filtering out the cells with a low number of genes detected, insufficient read counts and ERCC-dominated samples (Supplementary Fig. 2a-c; see Methods), we finally retained scRNA-seq data of 125 cells composed of 37,43 and 45 single cells in the 2-hr (young), 16-hr (early age) and 36-hr (late age) age groups, respectively, for further analysis. We also compared our scRNA-seq data to the only 2 available scRNA-seq datasets of $S$. cerevisiae published recently ${ }^{18,19}$. Our method yielded, on average, 2,202 genes detected per cell, which is comparable to the dataset from Gasch et al ${ }^{18}(2,202$ vs 2,392) with good accuracy and sensitivity, similar to the dataset from Nadal-Ribelles et al ${ }^{19}$ (Supplementary Fig. 2d-e; Supplementary Table 1).

\section{Cell-to-cell transcriptional variability during ageing in yeast. We sought to explore the} cell-to-cell transcriptional variability within different age groups using scRNA-seq data. Overall, we observed increased cell-to-cell transcriptional variability during ageing in yeast based on a correlation analysis in which the transcriptional variability was measured as the biological noise over the technical noise ${ }^{20}$ (Fig. 1b; see Methods). We verified this increase in cell-to-cell transcriptional variability alternatively using a quantitative statistical method ${ }^{21}$ and respectively identified 145, 312 and 524 highly variable genes (HVGs) with coefficients of variation $(\mathrm{CV})$ that were significantly higher than those of the ERCC spike-in reference within each age group (Supplementary Fig. 3a; see Methods). Interestingly, by Gene Ontology (GO) analysis of these HVGs using DAVID ${ }^{22}$, the biological processes of cellular iron ion homeostasis and siderophore transport were specifically found to be highly enriched in the 16-hr early age group with high statistical significance, implying an early heterogeneity during ageing in yeast with regard to iron transport (Supplementary Table. 
2).

Because all of the ageing single yeast cells analyzed did not have synchronized cell cycles, we wondered whether and to what extent the cell-to-cell transcriptional variability was associated with the cell cycle. We found that $19.3 \%, 12.8 \%$ and $15.5 \%$ of HVGs, respectively, among the 3 age groups were regarded as cell-cycle-regulated periodic genes $^{23}$ (Supplementary Fig. 3b). These results are consistent with a recent report of scRNA-seq in budding yeast that cell-cycle-regulated periodic genes were enriched in $\mathrm{HVGs}^{19}$. However, the trend of increased cell-to-cell transcriptional variability during ageing remained even when these cell-cycle-regulated periodic HVGs were removed from the 3 age groups (117, 272 and 443 HVGs remained, respectively; Supplementary Fig. 3b). We further confirmed this trend using principal component analysis (PCA). Regardless of whether the cell-cycle-regulated periodic genes were included in the scRNA-seq dataset used as input for the PCA or not, the 3 age groups were always successfully separated along the axis of first PCA component and were increasingly dispersed (Fig. 1c; Supplementary Fig. 3c); moreover, the top 30 genes based on the absolute loading values for the first PCA component always highly overlapped and were enriched in the biological process of cellular response to oxidative stress, which reflects ageing itself rather than the cell cycle (Supplementary Fig. 3d-e; Supplementary Table. 3). We also performed pseudotime analysis using Monocle ${ }^{24}$ and found that while the young cells (2-hr) were still very concentrated, the cells of the early age group (16-hr) had already become scattered along the trajectory (Fig. 1d; Supplementary Fig. 3f).

Global differential gene expression during ageing in yeast. In addition to exploring the cell-to-cell transcriptional variability within different age groups, the scRNA-seq data also allow us to globally investigate the differential gene expression between age groups. Thus, we conducted a pairwise comparison among the 3 age groups using DESeq2 ${ }^{25}$ (Supplementary Fig. 4a; see Methods). Obviously, more differentially expressed genes were found in the 36-hr late age group compared to the 2-hr group (Supplementary Fig. 4a, right panel; Supplementary Table. 4). The biological processes of oxidation-reduction and the oxidative stress response (OSR) were highly enriched in the 36-hr group (75 and 26 out of 551 genes, respectively), while translation and ribosome biogenesis were highly enriched in the 2-hr group (50 and 38 out of 138 genes, respectively) based on the GO analysis of biological process using DAVID ${ }^{22}$ (Fig. 1e, right panel). Moreover, 145 out of 551 genes that were highly expressed in the 36-hr late age group compared to the 2-hr group were enriched in mitochondrion as revealed by the GO analysis of cellular components (Fig. 1e, left panel; Supplementary Table. 4). 
The average normalized gene expression levels across age groups further demonstrated an age-dependent increase in oxidation-reduction, OSR and mitochondrion as well as a decrease in translation and ribosome biogenesis (Fig. 1f). Indeed, these transcriptome changes had already occurred in the 16-hr early age group. Although far fewer differentially expressed genes were found in the 16-hr early age group compared to the 2-hr group (Supplementary Fig. 4a, left panel), early signs of upregulation in oxidation-reduction and downregulation in ribosome biogenesis (15 out of 108 genes and 4 out of 10 genes, respectively) were observed (Supplementary Fig. 4b; Supplementary Table 4) . Notably, the global differentially expressed genes between age groups and their enriched GO categories from our scRNA-seq data were found to coincide well with a recent report of transcriptome changes during ageing in yeast ${ }^{9}$ and were even partially consistent with another proteome analysis of ageing in $C$. elegans ${ }^{26}$, although they were both based on bulk population analysis. These ageing associated GO categories analyzed by DAVID were also confirmed by ClusterProfiler ${ }^{27}$ (Supplementary Fig. 5a-f).

Weighted gene co-expression network analysis during ageing in yeast. To find the clusters of highly correlated genes during ageing in yeast, we performed a weighted gene co-expression network analysis (WGCNA) ${ }^{28-29}$, and generated 7 different gene coexpression modules (Fig. 2a; see Methods). Among these gene co-expression modules (Fig. 2b-d), we further identified 52 hub genes from 731 genes in the positively correlated modules which were upregulated during ageing (Supplementary Table. 5). These genes are mainly enriched in OSR and oxidation-reduction process by GO analysis using Metascape $^{30}$, and 5 of them are even involved in the longevity regulatory pathways, including HSP104, which is a molecular marker of ageing in yeast identified previously ${ }^{11,12}$ (Supplementary Fig. 6a; Supplementary Table. 5). 70 hub genes were identified from 410 genes in the negatively correlated modules which were downregulated during ageing and they are mainly enriched in ribosome biogenesis (Supplementary Fig. 6b). All these findings echo well the results of previous global differential gene expression analysis during ageing in yeast.

Differential gene expression between slow- and fast-dividing age subgroups. The number of genes detected per cell within age groups was found to be positively correlated with the generation, suggesting another facet to understand the heterogeneity of cell divisions during ageing in yeast, and the 16-hr and 36-hr age groups were thus split by their respective mean generation into slow-dividing (16-hr/S, 36-hr/S) and fast-dividing (16$\mathrm{hr} / \mathrm{F}, 36-\mathrm{hr} / \mathrm{F}$ ) subgroups (Fig. 3a, b; Supplementary Table. 1). Comparing the early age subgroups of $16-\mathrm{hr} / \mathrm{S}$ and $16-\mathrm{hr} / \mathrm{F}$ by DESeq2 ${ }^{25}$ with stringent statistical filtering yielded 29 differentially expressed genes, with 5 highly expressed and 24 weakly expressed in 16hr/S (Fig. 3c; Supplementary Table. 6). FIT3 and HAC1 are highly expressed in 16-hr/S. 
FIT3, together with FIT2 and FIT1, as facilitators of iron transport in yeast, encodes a cell wall mannoprotein ${ }^{31}$. These genes were reported to be induced upon iron deprivation or mitochondrial DNA loss ${ }^{32,33}$. HAC1 is a transcription factor that regulates the unfolded protein response (UPR), and interestingly, one of its regulatory targets is $F I T 3^{34,35}$. Indeed, FIT3 and HAC1 were not only highly expressed in 16-hr/S but also in 36-hr/S (Fig. 3d, e). Moreover, the gene expression of FIT3 and HAC1 negatively correlated with the age of single cells in the 16-hr age group $\left(R=-0.55,-0.38 ; P=1.3 \times 10^{-4}, 1.5 \times 10^{-2}\right)$ as well as the 36-hr group ( $R=-0.62,-0.44 ; P=5.6 \times 10^{-6}, 2.2 \times 10^{-3}$; Fig. 3f; Supplementary Fig. 7a; Supplementary Table. 6). Surprisingly, gene expression levels of several other iron transporters, including FIT2 and $F E T 3^{31}$, were also found to be negatively correlated with the generation of single cells in the 16-hr and 36-hr age groups (Supplementary Fig. 7b, c; Supplementary Table. 6). Finally, as single-gene deletions of FIT2 and FET3 were both reported to extend the lifespan in yeast ${ }^{4}$, we measured the RLS of yeast after deleting FIT3, and verified that this strain is long-lived as well (Fig. $3 \mathrm{~g}$ ). Collectively, these results clearly reveal a molecular marker of iron transport that can quantitatively indicate early heterogeneity during ageing in yeast, which might be mediated by mitochondrial DNA loss $^{33}$. This early ageing transcriptional signature can last until an advanced age and predict the lifespan.

Interestingly, we also revealed that 11 out of 24 genes expressed at low levels in 16-hr/S were enriched in mitochondrion, and these genes were also expressed at lower levels in 36-hr/S than in 36-hr/F (Fig. 3c-e; Supplementary Table. 6). This further suggests a relatively poor mitochondrial function in the slow-dividing cells. Among these 11 weakly expressed mitochondrial genes (Fig. 3c), COR1 is the core subunit of ubiquinolcytochrome $\mathrm{c}$ reductase which belongs to complexes III and COX4 is an important component of cytochrome $\mathrm{c}$ oxidase which belongs to complexes IV of the mitochondrial inner membrane electron transport chain. It has been reported that mutation of either COR 1 or COX 4 can cause a decrease in respiration, slow cell growth and even a shorter lifespan ${ }^{34-38}$. These 11 mitochondrial genes showed no overlap with the 145 mitochondrial genes that were globally upregulated during ageing (Fig. 1e and Fig. 3c, Supplementary Table. 4 and 6); in contrast, no significant differential expression of those 145 mitochondrial genes was observed between these two subgroups (Fig. 3e). These results successfully characterize divergent mitochondrial gene expression profiles between age groups and subgroups that would be masked in the bulk population analysis but can be identified by scRNA-seq.

The correlation analysis between the gene expression and the generation of single cells also resulted in genes that were positively correlated with generation in the 16-hr early age group are enriched in ribosome biogenesis (Supplementary Fig. 7d; Supplementary Table. 
6). This suggests a downregulation of at least some ribosome biogenesis genes during early ageing and it is mainly contributed by the cells from the slow-dividing age subgroup, which are inclined to be short-lived (Supplementary Fig. 7e). Meanwhile, genes enriched in translation, mitochondrial translation and glycolytic processes were positively correlated with generation in the 36-hr late age group (Supplementary Fig. 7f). This agrees with the differential gene expression analysis above, suggesting a relatively poor machinery of translation and mitochondrion in the slow-dividing age subgroups. In summary, all these results thoroughly characterize early and late heterogeneity during ageing in yeast at the single-cell transcriptome level.

\section{Temporal regulation of transcription factor (TF) between age subgroups. We further} investigated the regulatory variation in transcription factors (TFs) between age subgroups, analyzing 634 overlapping TF targets (gene clusters) based on reported studies on budding yeast ${ }^{18,39-43}$. To eliminate false positives, we performed stringent statistical analysis with three approaches (see Methods). First, we conventionally compared the median TF target expressions between age subgroups. This led to $16 \mathrm{TF}$ targets that were significantly activated in the 16-hr/F subgroups and $11 \mathrm{TF}$ targets in 36-hr/F compared to their counterparts, respectively (Supplementary Fig. 8a, b; Supplementary Table. 7). Then, we ran a Wilcoxon rank sum test comparing normalized gene expression levels of each set of TF targets to that of all other detected genes for each cell, taking $P<0.0001$ as the criterion, followed by intersection with TF targets derived from the conventional analysis. This led to 5 and 2 TF targets that were significantly activated in 16-hr/F and 36-hr/F, respectively (Fig. 4a; Supplementary Fig. 8c; Supplementary Table. 7). Subsequently, we employed correlation analysis between TF target expression and the generation of single cells in the 16-hr and 36-hr age groups, taking $\mathrm{P}<0.05$ as the criterion (Supplementary Fig. 9a, b; Supplementary Table. 7), followed by intersection with TF targets derived from the former two approaches.

Finally, YAP1 was found to be most significantly active in regulating the early age subgroup of 16-hr/F compared to 16-hr/S (Fig. 4b, c), although the other 4 TFs of ABF1, REB1, INO4 and TYE7 demonstrated a similar trend with less statistical significance (Supplementary Fig. 8d, e). Moreover, 2 TF targets of RPN4 were found to be most highly regulated at 36$\mathrm{hr} / \mathrm{F}$ compared to $36-\mathrm{hr} / \mathrm{S}$ (Fig. $4 \mathrm{~b}, \mathrm{c}$ ). YAP1 is involved in activating the transcription of antioxidant genes in response to oxidative stress ${ }^{44,45}$. The relatively high activation of YAP1 targets in the 16-hr/F early age subgroup suggests that the rapidly dividing single cells, which are inclined to be long-lived, may have a better defence system against oxidative stress than the slow-dividing cells. RPN4 is a TF that stimulates proteasome biogenesis for the degradation of damaged proteins ${ }^{46}$. The relatively high activation of RPN4 targets in the $36-\mathrm{hr} / \mathrm{F}$ late age but rapidly dividing subgroup supports the idea that proteasome 
capacity is critical to maintain the vigour and proteostasis of yeast cells, especially when approaching the end of life, as elevated RPN4 expression is essential for extending the RLS in yeast ${ }^{47}$. Altogether, these findings reveal early and late heterogeneity by distinctive temporal regulation of TFs during ageing in yeast, and combined with the aforementioned differential gene expression analysis between age groups and subgroups, we successfully depicted a landscape of ageing in yeast with unprecedented detail at single-cell resolution.

\section{Discussion}

Although transcriptome changes during ageing in yeast based on bulk population analyses have been reported ${ }^{8,9}$, such analyses at the single-cell level had not yet been performed. Here, we first identified an early heterogeneity of cell divisions during ageing in yeast by single-cell imaging and then developed and applied scRNA-seq for single-cell transcriptome analysis during ageing in yeast for the first time.

Using scRNA-seq technology, we overcame the difficulty of purifying the large number of old cells required for conventional transcriptome analysis during ageing in yeast. More importantly, by single-cell transcriptome analysis, we not only successfully recapitulated the results of the bulk population analysis but also teased out specific transcriptional features at the single-cell resolution that would otherwise be masked in a bulk population. For example, by scRNA-seq we revealed that while globally there were an age-dependent upregulation of many mitochondrial genes between age groups, a small number of different but important mitochondrial genes were significantly downregulated in the slow-dividing age subgroups compared to their fast-dividing counterparts. This provides novel and unprecedented insights into our understanding of the ageing process. Our results have unveiled the increased cell-to-cell transcriptional variability independent of the cell cycle and identified an early heterogeneity during ageing in yeast. This also coincides with recent reports of scRNA-seq in mouse immune cells and human pancreatic cells during ageing ${ }^{48,20}$.

By single-cell transcriptome analysis, we also identified a new molecular marker of iron transport that both indicates early heterogeneity during ageing in yeast and predicts lifespan. Remarkably, FIT3 together with several other iron transporter genes, such as FIT2 and FET3, had a negative correlation with the age of single yeast cells from both early and late timepoints. These genes are known to be induced upon iron deprivation or mitochondrial DNA loss ${ }^{32,33}$. Moreover, these genes can all extend the RLS in yeast when deleted $^{4}$ (Fig. 3g). Therefore, we propose a model in which early heterogeneity during ageing in yeast is associated with differential mitochondrial dysfunction that affects and is mediated by iron transport. And this model is partially supported by a report published 
recently, showing age-dependent heterogeneity via a FIT2 reporter that is correlated with vacuolar $\mathrm{pH}$, mitochondrial function and lifespan in sub-populations of yeast cells ${ }^{50}$. More evidence may be needed to further validate this model, and presently it remains challenging to disentangle the cause-effect relationships between mitochondrial dysfunction and early heterogeneity during ageing. However, we keep optimistic that these problems can be solved if the potential of modern single-cell technologies integrated with other new methods are fully employed.

Based on the scRNA-seq data and knowledge of TF targets in the budding yeast Saccharomyces cerevisiae ${ }^{18,39-43}$, we also explored TF regulation at the single cell level and found distinctive temporal regulation of TFs during ageing in yeast. YAP1 is a key TF responding to oxidative stress ${ }^{44,45}$. While it was highly activated in $16-\mathrm{hr} / \mathrm{F}$ compared to $16-$ $\mathrm{hr} / \mathrm{S}$ early age subgroup, no significant difference of its activities were observed between 36-hr/F and 36-hr/S late age subgroups (Fig. 4b, c), implicating its vital role during early ageing, which in turn affects overall lifespan. In contrast, RPN4, the TF essential for proteasome biogenesis and RLS extension ${ }^{46,47}$, was only prominently activated in $36-\mathrm{hr} / \mathrm{F}$ compared to 36-hr/S late age subgroup, suggesting a dramatic loss of proteostasis in the late age and slow-dividing subgroup ${ }^{49}$ (Fig. 4b, c; Supplementary Fig. 8a-c; Supplementary Fig. 9a, b). These findings point not only to early but also late heterogeneity during ageing in yeast, and provide novel insights into understanding the molecular mechanisms of ageing that will lead to therapeutics for healthy ageing in humans ultimately ${ }^{51}$.

\section{Acknowledgement}

We thank the BIOPIC sequencing platform at Peking University for the assistance of highthroughput sequencing experiments. This work was supported by Ministry of Science and Technology of China (2018YFA0108100), National Natural Science Foundation of China (21917802, 21525521), 2018 Beijing Brain Initiative (Z181100001518004), and Beijing Advanced Innovation Center for Genomics.

\section{Author contributions}

Y.Z. and Y.H. conceived and designed the project. Y.Z., J.W., Y.S., S.J., X.Z., and G.K.A conducted the experiments. Y.Z., J.W., B.K., Q.L., J.W., X.Z. and Y.H. analyzed the data. Y.Z., J.W., B.K., X.Z., and Y.H. wrote the manuscript with the help from all other authors. 
bioRxiv preprint doi: https://doi.org/10.1101/2020.09.04.282525; this version posted September 4, 2020. The copyright holder for this preprint (which was not certified by peer review) is the author/funder, who has granted bioRxiv a license to display the preprint in perpetuity. It is made available under aCC-BY-NC-ND 4.0 International license.

391 The authors declare no conflict of interest

392 


\section{Methods}

Strains and growth conditions. WT Saccharomyces cerevisiae in both BY4741 and BY4742 backgrounds were used for single-cell imaging analysis. The strain of Hsp104GFP was derived from the standard GFP strain library in WT BY4741 background. WT BY4742 background was used in scRNA-seq during aging. WT BY4741 background was used in the replicative lifespan assay of FIT3A. For single-cell imaging, the cells were grown in the YPD liquid media before and after loading into the microfluidic chips. For scRNA-seq during aging and replicative lifespan assay of FIT3A, the cells were grown on SD solid agar plates.

Single-cell imaging data analysis. The approach for single-cell imaging data analysis has been reported in detail elsewhere ${ }^{11}$. Yeast cell culture was grown in YPED at $30^{\circ} \mathrm{C}$ with OD600 of 0.5 before loading into the microfluidic device by a syringe connected to an automatically controlled peristaltic pump. The microfluidic device was mounted on a Nikon TE2000 time-lapsed microscope by a customized holder. Bright field images were taken once every 10 minutes throughout the whole life, and fluorescent images were taken once every 2 hours or 4 hours for measuring the HSP104-GFP level. The images were processed by Image J and MATLAB.

Dissection and isolation of single cells for RNA-seq. We first inoculated WT yeast cells onto a solid agar plate with SD media and followed a standard protocol of replicative lifespan assay by continual (no storage in the $4^{\circ} \mathrm{C}$ fridge overnight) manual microdissection ${ }^{15}$. At 3 time points ( $2 \mathrm{hr}, 16 \mathrm{hr}$ and $36 \mathrm{hr}$ after birth), single yeast aging cells from the plate were manually dissected and placed individually into a single tube prefilled with lysis buffer containing zymolyase $\left(3 \times 10^{-2} \mathrm{U} / \mu \mathrm{l}\right)$ for efficiently digesting the cell wall and external RNA control consortium (ERCC) spike-in (8000 molecules) for assessing technical noise, followed by immediate freeze in liquid nitrogen and then storage in a $-80^{\circ} \mathrm{C}$ freezer.

Library preparation for ScRNA-seq . After collecting all the single yeast aging cells, we performed scRNA-seq based on Smart-seq2 ${ }^{16,17}$ with fine optimization. To efficiently lyse the single yeast aging cell and avoid possible mRNA degradation, we vigorously vortexed the lysis tubes for $1 \mathrm{~min}$ in a cold room. Then we kept the lysis tubes at $30^{\circ} \mathrm{C}$ for $10 \mathrm{~min}$, followed by $3 \mathrm{~min}$ at $72^{\circ} \mathrm{C}$. Subsequently, we added the RT reaction mix (RT-buffer and Invitrogen SuperScript II ) for reverse transcription. Reverse transcription was carried out at $42^{\circ} \mathrm{C}$ for 90 min first, followed by 12 rounds of temperature cycling between $50^{\circ} \mathrm{C}$ and 
down to $4^{\circ} \mathrm{C}$. The oligo-dT and TSO primers used here were biotinylated to avoid potential production of excessive primer dimers and concatamers. After RT, the cDNA were amplified between 20 25 cycles using KAPA HiFi enzyme. After cDNA amplification, the samples were purified using Agencourt AMPure XP beads at $0.8 \mathrm{X}$ bead concentration and quantified using Qubit Hs Assay (Life Technologies). We also checked the samples by a fragment analyzer to confirm the clean peak at $\sim 1.7 \mathrm{~kb}$ before subsequent processing. 1 2 ng of cDNA was subjected to a tagmentation-based protocol (Vazyme TruePrep Kit) with $10 \mathrm{~min}$ at $55^{\circ} \mathrm{C}$ and dual index amplification for the library with 8 12 cycles. The final libraries were purified twice using AMPure XP beads at $0.8 \mathrm{X}$ bead concentration and resuspended in 15 20 $\mu$ l elution buffer. Libraries were then quantified using Qubit Hs Assay before pooling for sequencing. Sequencing was performed in paired-end mode using Illumina NextSeq.

scRNA-seq data pre-processing and filtering. Paired-end reads were mapped to the S288c Saccharomyces cerevisiae genome R64 version (www.yeastgenome.org) with ERCC spike-in sequences added using HISAT2 (version 2.1.0). Resulting bam files were sorted and indexed using samtools (version 1.1). Final read counts mapped to genes were extracted using FeatureCounts. Sequenced single yeast aging cells were removed from the analysis if they have $<1000$ genes detected and 40,000 total mapped reads per cell, or if the proportion of ERCC spike-ins to total-mapped reads was $>0.74$. After filtering, a scRNA-seq data set with 125 single yeast aging cells was used for the subsequent analysis.

Normalization. Unless noted, normalization of raw read counts was done using the DESeq2 ${ }^{25}$ package (v.1.22.2) in R. The size factor was computed by a formula embedded in DESeq2 for each cell based on the raw read counts matrix of all samples. Then these size factors were applied for normalizing different cells and finally the gene expression values are presented in the $\log _{2}$ space ( $\log _{2}$ NormCounts).

\section{Estimation of cell-to-cell transcriptional variability and identification of highly} variable genes. We used two methods to estimate the cell-to-cell transcriptional variability during aging in yeast. The first was a correlation based method modified from Enge, M. et $a^{20}$, where the transcriptional noise was expressed as biological variation over technical variation. First, we calculated the biological variation $b_{i j}=1-\operatorname{cor}\left(x_{i j}, u_{i}\right)$, where $u_{i}$ was the mean gene expression vector for the single cells in age group of $\mathrm{i}(2 \mathrm{hr}, 16 \mathrm{hr}$ and $36 \mathrm{hr}$ ), and $x_{i j}$ was the gene expression vector of cell $j$ in the age group of $i$. Next, we calculated the corresponding technical variation $t_{i j}=1-\operatorname{cor}\left(x^{\text {contr }}\right.$ ij, $\left.u^{\text {contr }}\right)$ where $x^{\text {contr }}$ ij and $u^{\text {contr }}$ are the expression vector and mean expression vector of the ERCC spike-in controls. Finally the measurement of $b_{i j} / t_{i j}$ which reflected the biological noise as a fraction of technical noise 
for each cell was used for boxplot across different age groups as shown in Fig.1b. The second method was based on quantitative statistics reported previously ${ }^{21}$ (see Supplementary Note 6 of Brennecke et $\mathrm{al}^{21}$ for details of the statistical model). Briefly, to infer the genes that were highly variable within each age group, a linear regression model was applied to fit the relationship between the squared coefficient of variation $\left(\mathrm{CV}^{2}\right)$ and the mean expression of ERCC spike-ins, and only genes with biological squared coefficient of variation $>0.25\left(\mathrm{CV}^{2}>0.25\right)$ and $\mathrm{FDR}<0.1$ after multiple testing correction were regarded as HVGs.

Differential gene expression and GO analysis. The differential gene expression analysis between pairwise age groups and subgroups was based on DESeq2 ${ }^{25}$ with default parameters, taking $\log _{2} \mathrm{FC}>1$ and adjusted $\mathrm{P}$ value $<0.05$ as significant. $\mathrm{GO}$ analysis of these differentially expressed genes was performed by functional annotation tool of DAVID 22 that classify the ontology of each gene into biological process or cellular component. The GO term enrichment results derived from DAVID were further verified alternatively by the $\mathrm{R}$ package of ClusterProfiler ${ }^{27}$.

Weighted gene co-expression network analysis. WGCNA ${ }^{28-29}$ was performed on normalized gene expression data from DESeq $2^{25}$, using 2498 genes, which are selected by removing unclassified genes (grey module) from the first round of WGCNA ${ }^{28-29}$. Then the second round WGCNA ${ }^{29-30}$ was performed following the standard process. Briefly, the topological overlap matrix (TOM) was constructed with a soft Power and was set to 2 . The hub genes for each module were identified as module membership based group trait $>$ 0.65 and gene significance $>0.2$.

\section{Statistical analysis of regulation of transcription factor between age subgroups. To} identify transcription factors with distinct regulation between age subgroups, 3 statistical approaches were applied stringently. The first one was to conventionally comparison of TF targets expression between age subgroups. The TF targets expression was defined as the averaged normalized expression of each set of TF targets for each cell. And we took $\log _{2} \mathrm{FC}$ (FoldChange) of median TF targets expression between age subgroups $>1\left(\log _{2} \mathrm{FC}>1\right)$ and a welch $t$ test $P$ value $<0.01$ as significant, which resulted in 16 and 11 TF targets respectively that were significantly activated in the age subgroups of $16-\mathrm{hr} / \mathrm{F}$ and $36-\mathrm{hr} / \mathrm{F}$ compared to their counterparts (Supplementary Fig. 8a, b; Supplementary Table. 6). The second one was to further run a Wilcoxon rank sum test for each single cell that compare internally the normalized gene expression levels of each set of TF targets to all other detected genes for that cell, taking $\mathrm{P}<0.0001$ as criterion (indicated as regulon activity "on" ), followed by intersection with TF targets derived from the first approach. This 
approach was similar with that from Gasch et $a^{18}$. The last one was to correlate the TF targets expression with the generation of single cells in the age groups of 16-hr and 36-hr respectively, taking $\mathrm{P}<0.05$ as criterion, followed by intersection with TF targets derived from the former two approaches to avoid potential false positive results.

PCA analysis. Raw read counts matrix with or without cell-cycle-regulated periodic genes ${ }^{23}$ were used as inputs for PCA by Seurat ${ }^{52}$. When the cell-cycle-regulated periodic genes were included, Seurat generates 631 common variable genes of all 125 single yeast aging cells, whose normalized read counts are further applied for PCA. When the cellcycle-regulated periodic genes were excluded, Seurat generated 599 common variable genes of all 125 single yeast aging cells for PCA.

\section{Data availability}

scRNA-seq data generated in this study has been uploaded to Gene Expression Omnibus under accession number xxxxxx.

\section{References}

1. Mortimer, R. K. \& Johnston, J. R. Life Span of Individual Yeast Cells. Nature 183, 1751-1752 (1959).

2. Kaeberlein, M., Burtner, C. R. \& Kennedy, B. K. Recent Developments in Yeast Aging. PLOS Genetics 3, e84 (2007).

3. Denoth Lippuner, A., Julou, T. \& Barral, Y. Budding yeast as a model organism to study the effects of age. FEMS Microbiol Rev 38, 300-325 (2014).

4. McCormick, Mark A. et al. A Comprehensive Analysis of Replicative Lifespan in 4,698 Single-Gene Deletion Strains Uncovers Conserved Mechanisms of Aging. Cell Metabolism 22, 895-906 (2015).

5. Smeal, T., Claus, J., Kennedy, B., Cole, F. \& Guarente, L. Loss of Transcriptional Silencing Causes Sterility in Old Mother Cells of S. cerevisiae. Cell 84, 633-642 (1996).

6. Lesur, I. \& Campbell, J. L. The transcriptome of prematurely aging yeast cells is similar to that of telomerase-deficient cells. Mol Biol Cell 15, 1297-1312 (2004).

7. Lindstrom, D. L. \& Gottschling, D. E. The mother enrichment program: a genetic system for facile replicative life span analysis in Saccharomyces cerevisiae. Genetics 183, 413-422 (2009).

8. Janssens, G. E. et al. Protein biogenesis machinery is a driver of replicative aging in yeast. elife 4, e08527 (2015). 

and chromatin landscapes of aging yeast. eLife 7, e39911 (2018).

10. Zhang, Y., Gao, J., Huang, Y. \& Wang, J. Recent Developments in Single-Cell RNA-Seq of Microorganisms. Biophys J 115, 173-180 (2018).

11. Zhang, Y. et al. Single Cell Analysis of Yeast Replicative Aging Using a New Generation of Microfluidic Device. PLOS ONE 7, e48275 (2012).

12. Xie, Z. et al. Molecular phenotyping of aging in single yeast cells using a novel microfluidic device.

544 Aging Cell 11, 599-606 (2012).

13. Xie, Z. et al. Early Telomerase Inactivation Accelerates Aging Independently of Telomere Length. Cell 160, 928-939 (2015).

14. Yang, J., Wang, Z., Liu, X., Li, H. \& Ouyang, Q. Yeast replicative aging leads to permanent cell cycle arrest in G1 effectuated by the start repressor Whi5. bioRxiv, 353664 (2018). the Budding Yeast. JoVE, e1209 (2009). Methods 10, 1096-1098 (2013).

18. Gasch, A. P. et al. Single-cell RNA sequencing reveals intrinsic and extrinsic regulatory heterogeneity in yeast responding to stress. PLOS Biology 15, e2004050 (2017). 19. Nadal-Ribelles, M. et al. Sensitive high-throughput single-cell RNA-seq reveals within-clonal transcript correlations in yeast populations. Nature Microbiology 4, 683-692 (2019).

20. Enge, M. et al. Single-Cell Analysis of Human Pancreas Reveals Transcriptional Signatures of Aging and Somatic Mutation Patterns. Cell 171, 321-330 (2017). Methods 10, 1093-1095 (2013). Biology 4, P3 (2003).

23. Granovskaia, M. V. et al. High-resolution transcription atlas of the mitotic cell cycle in budding yeast. Genome Biology 11, R24 (2010). pseudotemporal ordering of single cells. Nature Biotechnology 32, 381-386 (2014). 
25. Love, M. I., Huber, W. \& Anders, S. Moderated estimation of fold change and dispersion for RNA-seq data with DESeq2. Genome Biology 15, 550 (2014).

26. Walther, Dirk M. et al. Widespread Proteome Remodeling and Aggregation in Aging C. elegans. Cell 161, 919-932 (2015). 27. Yu, G., Wang, L.-G., Han, Y. \& He, Q.-Y. clusterProfiler: an R Package for Comparing Biological Themes Among Gene Clusters. OMICS: A Journal of Integrative Biology 16, 284-287 (2012).

28. Zhang, B., Horvath, S. A General Framework for Weighted Gene Co-Expression Network Analysis. Statistical Applications in Genetics and Molecular Biology. 4, (2005). PMID: 16646834.

29. Langfelder, P., Horvath, S. WGCNA: an R package for weighted correlation network analysis. BMC Bioinformatics. 9: 559 (2008).

579 30. Zhou, Y., Metascape provides a biologist-oriented resource for the analysis of systems-level datasets.

$580 \quad$ Nature Communications 10, 1523 (2019).

581 31. Protchenko, O. et al. Three cell wall mannoproteins facilitate the uptake of iron in 582 Saccharomyces cerevisiae. J Biol Chem 276, 49244-49250 (2001).

583 32. Foury, F. \& Talibi, D. Mitochondrial control of iron homeostasis. A genome wide analysis of gene expression in a yeast frataxin-deficient strain. J Biol Chem 276, 7762-7768 (2001).

33. Veatch, J. R., McMurray, M. A., Nelson, Z. W. \& Gottschling, D. E. Mitochondrial Dysfunction Leads to Nuclear Genome Instability via an Iron-Sulfur Cluster Defect. Cell 137, 1247-1258 (2009).

34. Cox, J. S. \& Walter, P. A Novel Mechanism for Regulating Activity of a Transcription Factor That 588 Controls the Unfolded Protein Response. Cell 87, 391-404 (1996).

589 35. Hu, Z., Killion, P. J. \& Iyer, V. R. Genetic reconstruction of a functional transcriptional regulatory network. Nature Genetics 39, 683-687 (2007).

592 Q biosynthesis, respiratory electron transport, and antioxidant function in Saccharomyces 593 cerevisiae. Biochim Biophys Acta 1831, 776-791 (2013).

594 37. Herrmann JM, Funes S. Biogenesis of cytochrome oxidase-sophisticated assembly lines in the 595 mitochondrial inner membrane. Gene 354, 43-52 (2005).

596 38. Marek A, Korona R. Restricted pleiotropy facilitates mutational erosion of major life-history 597 traits. Evolution 67, 3077-86 (2013).

598 39. Huebert, D. J., Kuan, P.-F., Keleş, S. \& Gasch, A. P. Dynamic Changes in Nucleosome Occupancy Are 599 Not Predictive of Gene Expression Dynamics but Are Linked to Transcription and Chromatin Regulators. 600 Molecular and Cellular Biology 32, 1645-1653 (2012).

601 40. Harbison, C. T. et al. Transcriptional regulatory code of a eukaryotic genome. Nature 431, 99-104, 602 (2004). 
41. Maclsaac, K. D. et al. An improved map of conserved regulatory sites for Saccharomyces cerevisiae. BMC Bioinformatics 7, 113, (2006).

42. Venters, B. J. et al. A Comprehensive Genomic Binding Map of Gene and Chromatin Regulatory Proteins in Saccharomyces. Molecular Cell 41, 480-492 (2011).

43. Abdulrehman, D. et al. YEASTRACT: providing a programmatic access to curated transcriptional regulatory associations in Saccharomyces cerevisiae through a web services interface. Nucleic acids research. 39, (Database issue):D136-D140 (2011).

44. Toone, W. M. \& Jones, N. AP-1 transcription factors in yeast. Curr Opin Genet Dev. 9, 55-61 (1999).

45. Temple, M. D. et al. Complex cellular responses to reactive oxygen species. Trends Cell Biol 15, 319326 (2005).

46. Xie, Y. \& Varshavsky, A. RPN4 is a ligand, substrate, and transcriptional regulator of the $26 \mathrm{~S}$ proteasome: a negative feedback circuit. Proc Natl Acad Sci USA 98, 3056-3061 (2001).

47. Undine, K. et al. Elevated Proteasome Capacity Extends Replicative Lifespan in Saccharomyces cerevisiae. PLoS Genetics. 7, e1002253 (2011).

48. Martinez-Jimenez, C. P. et al. Aging increases cell-to-cell transcriptional variability upon immune stimulation. Science 355, 1433-1436 (2017).

49. Hipp, MS., Kasturi, P., Hartl, FU. The proteostasis network and its decline in ageing. Nat Rev Mol Cell Biol. 20, 421-435 (2019).

50. Chen, Kenneth L. et al. Loss of vacuolar acidity results in iron-sulfur cluster defects and divergent homeostatic responses during aging in Saccharomyces cerevisiae. GeroScience. 42, 749-764 (2020).

51. Judith, Campisi. et al. From discoveries in ageing research to therapeutics for healthy ageing. Nature. 571, 183-192 (2019).

52. Butler, A., Hofman, P., Smibert, P., Papalexi, E. \& Satija, R. Integrating single-cell transcriptomic data across diferent conditions, technologies, and species. Nat. Biotechnol. 36, 411-420 (2018). 


\section{Figure legends}

Fig.1 | Cell-to-cell transcriptional variability and global differential gene expression during ageing in yeast. a, Schematic of the workflow of scRNA-seq during aging in yeast. Each single yeast ageing cell (indicated as gray ellipse in the dashed area) was manually isolated at 2-hr, 16-hr or 36-hr after birth, and then placed individually into a single tube prefilled with lysis buffer, followed by modified and optimized Smart-seq2 ${ }^{16,17}$. b, Boxplot showing an increased cell-to-cell transcriptional variability during aging in yeast based on a correlation analysis where the transcriptional variability was measured as biological noise over the technical noise (see Methods). Boxes indicated the first and third quartiles, separated by median line. Whiskers indicated last values within $1.5 \mathrm{x}$ the interquartile range for the box; Wilcoxon P values were also shown. c, PCA plot of single cells $(n=125)$ from different age groups (no cell-cycle-regulated periodic genes included as input for PCA). The distribution of single yeast aging cells in the 36-hr late age group was more scattered than that of 2-hr age group and 16-hr early age group, which reflected an increased cellto-cell transcriptional variability. $\mathbf{d}$, Pseudotime trajectory of single cells $(n=125)$ from different age groups (no cell-cycle-regulated periodic genes included as input). The youngest 2-hr age group was very concentrated, whereas the 16-hr early age group and 36-hr late age group were very scattered. e, (left) Heatmap of normalized gene expression of 551 upregulated and 138 downregulated genes in the 36-hr age group compared to 2$\mathrm{hr}$ age group, across different age groups. The purple bar indicated 145 mitochondrial genes that were highly expressed in the 36-hr late age group. (right) Significance of GO terms of biological processes (BP) in upregulated and downregulated genes respectively $\left(-\log _{10} P\right) . \mathbf{f}$, Boxplot of the average normalized expression of significantly upregulated and downregulated gene categories identified in $\mathbf{e}$, across different age groups. Each black dot in $\mathrm{f}$ represented a single cell. ${ }^{* *} \mathrm{p}<5.5 \times 10^{-7}$, ${ }^{* \star *} \mathrm{p}<4.2 \times 10^{-9}$, ${ }^{* * * *} \mathrm{p}<1.6 \times 10^{-13}$, from Wilcoxon rank sum test.

Fig.2 | Weighted gene co-expression network analysis during ageing in yeast. a, Dendrogram showing the gene co-expression network constructed using WGCNA. The color bar labeled as "Module" beneath the dendrogram represents the module assignment of each gene. We totally identified 7 modules. b, Module-trait relationship shows that the turquoise module is most positively while the blue module is most negatively correlated with the traits of Group and Generation of the single yeast cells. The upper number within cell represents correlation coefficient and number within brackets refers to the p-value. c and $\mathbf{d}$, Heatmap and barplot showing genes in the turquoise module are upregulated while genes in the blue module are downregulated during ageing in yeast. The rows of heatmap represent gene expression within the corresponding module. The columns of heatmap and barplot refer to the sample.

Fig.3 | Differential gene expression between slow- and fast-dividing age subgroups. a, (left) Correlation of the number of genes detected and the generation of single cells in 
the 16-hr early age group. Each red dot represented a single cell with the number of genes detected and its generation at $16 \mathrm{hr}$. Blue line was a linear fit with gray area indicating 0.95 confidence interval; correlation coefficient $(R)$ and $P$ value $(P)$ were also shown. The dashed line indicated the mean generation. The plot showed a positive correlation between the number of genes detected and the generation at $16 \mathrm{hr}$ among individual cells. (right) Boxplot of generation between early age subgroups $16-\mathrm{hr} / \mathrm{S}$ and $16-\mathrm{hr} / \mathrm{F}$ that were split by the mean generation of $16-$ hr early age group; Wilcoxon $P$ value was shown. b, (left) Correlation of the number of genes detected and the generation of single cells at 36-hr late age group and (right) Boxplot of generation between late age subgroups 36-hr/S and 36$\mathrm{hr} / \mathrm{F}$ that were split by the mean generation of 36 -hr late age group, plotted same as in a. Note: The cells with the number of genes below 1000 plotted in both $\mathbf{a}$ and $\mathbf{b}$ were discarded in the rest analysis. c, Differential gene expression analysis between early age subgroups $16-\mathrm{hr} / \mathrm{S}$ and $16-\mathrm{hr} / \mathrm{F}$. The heatmap showed normalized gene expression of statistically significant $\left(\log _{2}|F C|>1\right.$ and $\left.P_{a d j}<0.05\right)$ upregulated and downregulated genes in early age subgroup $16-\mathrm{hr} / \mathrm{S}$ compared to $16-\mathrm{hr} / \mathrm{F}$, across different age subgroups. d, Boxplots of normalized expression of significant differentially expressed genes of FIT3, $H A C 1$, and gene category of mitochondrion identified in $\mathbf{c}$ across different age groups. e, Boxplots of normalized expression of significant differentially expressed genes of FIT3, $H A C 1$, and gene categories of mitochondrion respectively identified in $\mathbf{c}$ and Fig. 1e across different age subgroups. Each black dot in $\mathbf{d}$ and $\mathbf{e}$ represented a single cell. ${ }^{*} p$ and ${ }^{* *} p<$ $0.05,{ }^{* * *} p<0.01,{ }^{* * * *} p<6.1 \times 10^{-5}$, and "ns" means not significant, from Wilcoxon rank sum test. $\mathbf{f}$, Correlation of normalized gene expression of FIT3 and the generation of single cells in the 16-hr early age group and 36-hr late age group, respectively. Each red dot represented a single cell. Blue line was a linear fit with gray area indicating 0.95 confidence interval; correlation coefficient $(R)$ and $P$ value $(P)$ were also shown. The plot showed a negative correlation for both age groups. $g$, Survival curve of WT and FIT3 $\Delta$. The number in the parenthesis represented the mean RLS and " $n$ " indicated the number of cells assayed for RLS of each strain.

\section{Fig.4 | Temporal regulation of transcription factor (TF) between age subgroups. a,} Heatmap showing differential expression of 5 transcription factor targets in the early age subgroup of 16-hr/F compared to 16-hr/S, and 2 transcription factor targets in the late age subgroup of $36-\mathrm{hr} / \mathrm{F}$ compared to $36-\mathrm{hr} / \mathrm{S}$, based on first two statistical criteria (see Methods). $\mathbf{b}$ and $\mathbf{c}$, Boxplots of differential expression of YAP1 targets that were highly expressed in the early age subgroup of 16-hr/F compared to 16-hr/S, and 2 RPN4 targets that were highly expressed in the late age subgroup of 36-hr/F compared to $36-\mathrm{hr} / \mathrm{S}$ identified by 3 stringent statistical approaches (see Methods), across different age groups and subgroups, respectively. Each black dot in $\mathbf{b}$ and $\mathbf{c}$ represented a single cell. ${ }^{*} \mathrm{p}<$ $0.05,{ }^{* *} p<0.01,{ }^{* * *} p<1 \times 10^{-3},{ }^{* * *} p<1 \times 10^{-4}$, and "ns" means not significant, from Wilcoxon rank sum test.

\section{Supplementary Fig.1 | Early heterogeneity of cell divisions during ageing in yeast. a,}


The distribution of generation at $8 \mathrm{hr}, 12 \mathrm{hr}$ and $16 \mathrm{hr}$ after birth of single yeasts respectively $(n=67)$. The plot showed the heterogeneity of cell divisions occurs early during ageing in yeast as indicated by the mean (Mean) and standard deviation (Std) of generation in the figure. $\mathbf{b}$, The lifespan was plotted against the generation at $8 \mathrm{hr}, 12 \mathrm{hr}$ and $16 \mathrm{hr}$ after birth of single yeasts respectively. It show a positive correlation. c, The HSP104-GFP level was plotted against the generation at $8 \mathrm{hr}, 12 \mathrm{hr}$ and $16 \mathrm{hr}$ after birth of single yeasts respectively. It show a negative correlation. Each red dot in $\mathbf{b}$ or $\mathbf{c}$ represented a single cell with its generation and its final lifespan or HSP104-GFP level, while blue line was a linear fit with gray area indicating 0.95 confidence interval; correlation coefficient $(R)$ and $P$ value $(P)$ were also shown.

\section{Supplementary Fig.2 | Data filtering and technical assessment of scRNA-seq. a, The} number of raw read counts plotted against the number of genes detected per cell between different age groups. $\mathbf{b}$, The ERCC ratio plotted against the number of genes detected per cell between different age groups. c, The ERCC ratio plotted against the number of raw read counts per cell between different age groups. Each dot in a-c represented a single cell with color indicating the age group or filtering status it belonged to ( $n=136$ cells). $d$ and e were mean normalized read counts and detection rate (the probability to have a read count number more than 0 ) plotted against the absolute number of RNA molecules per cell for each of the 92 ERCC RNA spike-in across all the single yeast aging cells that were filtered ( $n=125$ cells).

\section{Supplementary Fig.3 | Identification of HVGs within different age groups with or} without cell-cycle-regulated periodic genes. a, Squared coefficients of variation were plotted against the average normalized read counts for each cell within different age groups with cell-cycle-regulated periodic genes included. A gene was considered as HVG if the coefficient of biological variation was more than 0.5 (with the false discovery rate of 0.1 ). Red line represented the technical noise fit estimated by the ERCC spike-in RNA ${ }^{21}$ (see Methods). Endogenous genes, ERCC and HVGs were shown in black, green and magenta dots respectively. $\mathbf{b}$, Venn diagrams of HVGs within different age groups and the putative cell-cycle-regulated periodic genes. The increased cell-to-cell transcriptional variability during ageing still existed even excluding these cell-cycle-regulated periodic HVGs from 3 age groups. c, PCA plot of single cells $(n=125)$ from different age groups (with cell-cycleregulated periodic genes included as input for PCA). The 3 age groups were segregated along the first PCA component successfully. $\mathbf{d}$, Visualized plots of top 30 genes by absolute loading values for the first PCA component, with or without cell-cycle-regulated periodic genes included as input for PCA. e, Venn diagrams of the genes with top 30 genes by absolute loading values for the first PCA component, with or without cell-cycle-regulated periodic genes included as input for PCA, overlapped with putative cell-cycle-regulated periodic genes. $\mathbf{f}$, Pseudotime trajectory of single cells $(n=125)$ from different age groups (with cell-cycle-regulated periodic genes included as input). The youngest 2-hr age group was very concentrated, whereas the 16-hr early age group and 36-hr late age group were 
very scattered in order.

Supplementary Fig.4 | Differential gene expression between age groups. a, Volcano plot of global differential gene expression analysis between different age groups using DESeq2 (see Methods). The criteria for statistical significance were $\log _{2}$ foldchange of absolute normalized gene expression more than $1\left(\log _{2}|\mathrm{FC}|>1\right)$ and adjusted $P$ value less than $0.05\left(P_{\text {adj }}<0.05\right)$. b, Boxplots of the average normalized expression of typical upregulated and downregulated gene categories identified in the early age group of $16 \mathrm{hr}$ compared to $2 \mathrm{hr}$, across different age groups. Each black dot in $\mathbf{b}$ represented a single cell. ${ }^{*} p<0.05$ and ${ }^{* * *} p<1.4 \times 10^{-10}$ from Wilcoxon rank sum test.

Supplementary Fig.5 | GO enrichment analysis between age groups. a-f were GO enrichment analysis of differentially expressed genes from the pairwise comparison of 3 age groups using the $R$ package clusterProfiler ${ }^{27}$ (see Methods). The number of genes in the enriched $G O$ category was indicated by the size of the dot while the adjusted $P$ value was indicated by the color of the dot.

\section{Supplementary Fig.6 | GO analysis of hub genes of ageing related co-expression} gene module identified by WGCNA. a, GO terms of 52 hub genes of turquoise module. These hub genes were upregulated during ageing in yeast and are mainly enriched in OSR, oxidation-reduction process and even longevity regulating pathway. b, GO terms of 70 hub genes of blue module. These hub genes were downregulated during ageing in yeast and are mainly enriched in ribosome biogenesis.

\section{Supplementary Fig.7 | Correlation of gene expression and the generation of single} cells in the early and late age groups. a-c, Normalized gene expression of HAC1, FET3 and FIT2 plotted against the generation of single cells in the 16-hr early age group and 36hr late age group, respectively. Each red dot represented a single cell with the respective normalized gene expression and its generation, while blue line was a linear fit with gray area indicating 0.95 confidence interval; correlation coefficient $(R)$ and $P$ value $(P)$ were also shown. They all showed negative correlation with statistical significance $(P<0.05)$ except the FIT2 at $16 \mathrm{hr}(\mathrm{P}=0.14)$. $\mathrm{d}$, Pearson correlation of normalized gene expression with the generation of single cells in the early age group of $16 \mathrm{hr}$, taking $P<0.05$ as significant. The biological process of iron transport was enriched as negatively correlated while the ribosome biogenesis positively correlated. e, Boxplots of the average normalized expression of gene category of ribosome biogenesis identified in $\mathbf{d}$, across different age groups and subgroups. Each black dot in e represented a single cell. ${ }^{* * *} \mathrm{p}<2.7 \times 10^{-4}$, ${ }^{* * *} \mathrm{p}$ $<3.3 \times 10^{-6}$, and "ns" means not significant, from Wilcoxon rank sum test. f, Pearson correlation of gene expression with the generation of single cells in the 36-hr late age group, taking $\mathrm{P}<0.05$ as significant. The biological process of iron transport was enriched as 
negatively correlated while the translation, mitochondrial translation and glycolytic process positively correlated.

Supplementary Fig.8 | Distinct regulation of TF between age subgroups. a, 16 TF targets that were significantly activated in the early age subgroup of $16-\mathrm{hr} / \mathrm{F}$ by conventional comparison of median TF targets expressions to 16 -hr/S, taking $\log _{2} \mathrm{FC}>1$ and $P<0.01$ as significant. $\mathbf{b}, 11 \mathrm{TF}$ targets that were significantly activated in the late age subgroup of 36-hr/F by conventional comparison of median TF targets expressions to 36$\mathrm{hr} / \mathrm{S}$, taking $\log _{2} \mathrm{FC}>1$ and $\mathrm{P}<0.01$ as significant. c, The significantly activated TF targets in $16-h r / F$ and $36-h r / F$ in contrast to their counterparts were further narrowed down to 5 and 2 (highlighted in red in $\mathbf{a}$ and $\mathbf{b}$ ) respectively by Wilcoxon rank sum test comparing normalized gene expression levels of each set of TF targets to that of all other detected genes for each cell, taking $\mathrm{P}<0.0001$ as the criterion and indicated as "on" of the regulon activity. $\mathbf{d}$ and $\mathbf{e}$ are boxplots of differential expression of 4 other TF targets that were highly expressed in the early age subgroup of 16-hr/F compared to 16-hr/S identified by the first two stringent statistical approaches (see Methods), across different age groups and subgroups, respectively. Each black dot in $\mathbf{d}$ and e represented a single cell. ${ }^{*} \mathrm{p}<0.05$, ${ }^{* *} \mathrm{p}$ $<0.01,{ }^{* * *} p<2.8 \times 10^{-4},{ }^{* * * *} p<7.1 \times 10^{-5}$, and "ns" means not significant, from Wilcoxon rank sum test.

\section{Supplementary Fig.9 | Correlation of TF targets expression with the generation of} single cells in the early and late age groups. a, Pearson correlation of median TF targets expression with the generation of single cells in the 16-hr early age group, taking $P<0.05$ as significant. The expression of YAP1 targets was found to be most positively correlated. b, Pearson correlation of median TF targets expression with the generation of single cells in the 36-hr late age group, take $\mathrm{P}<0.05$ as significant. The expression of 2 RPN4 targets identified by previous two statistical approaches also positively correlated with the generation of single cells in the 36-hr late age group. 
a Align 2-hr pick

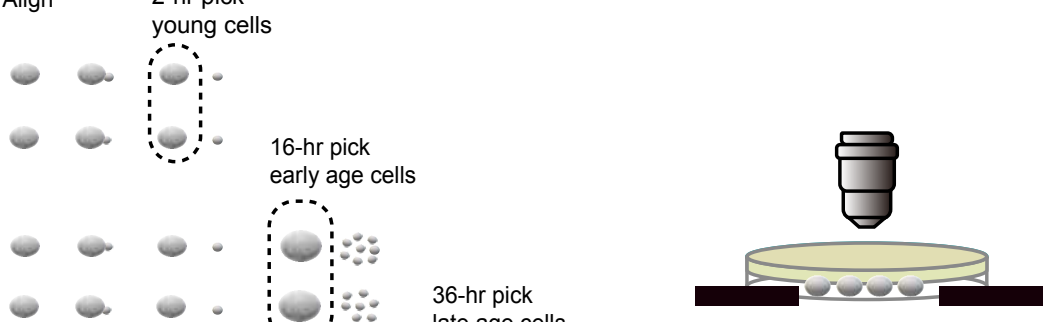

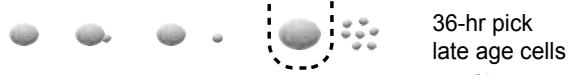

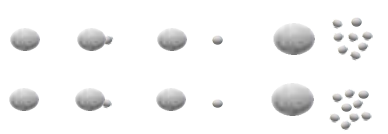

Sampling Strategy

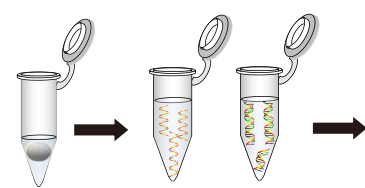

Lysis RT \& Amplification

Manual Dissection of Single Yeast Aging Cells
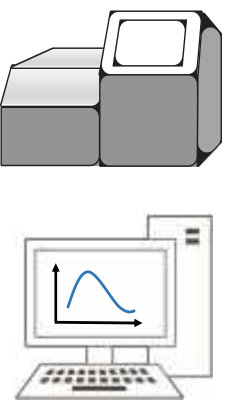

Sequencing \& Data Analysis b

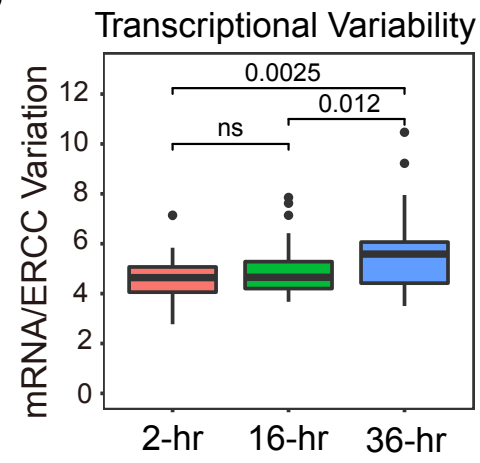

C

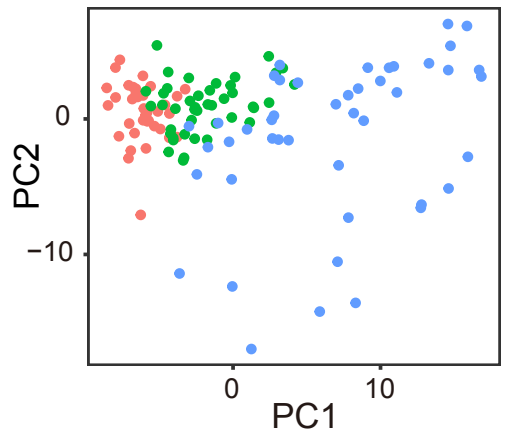

d

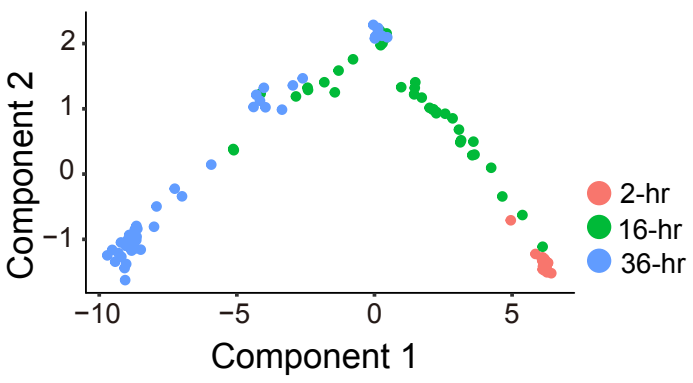

e

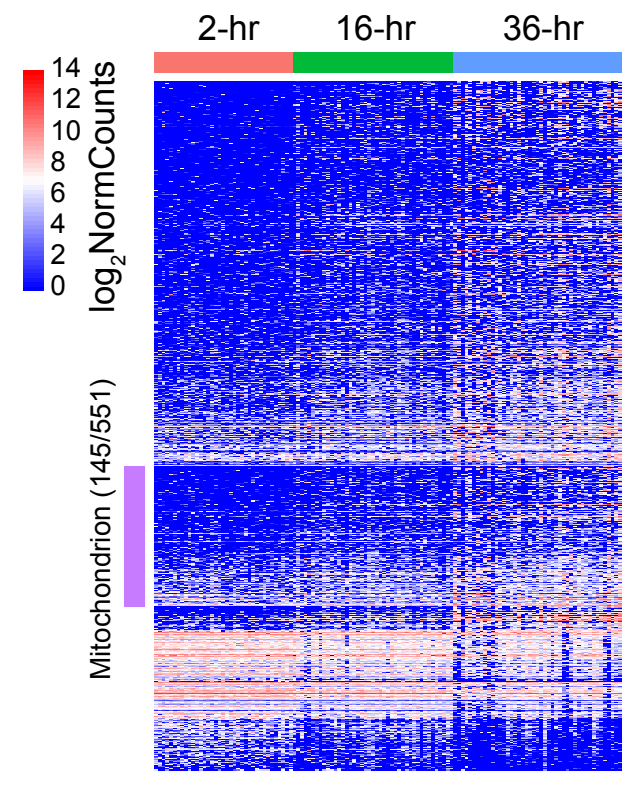

36-hr vs 2-hr GO term enrichment

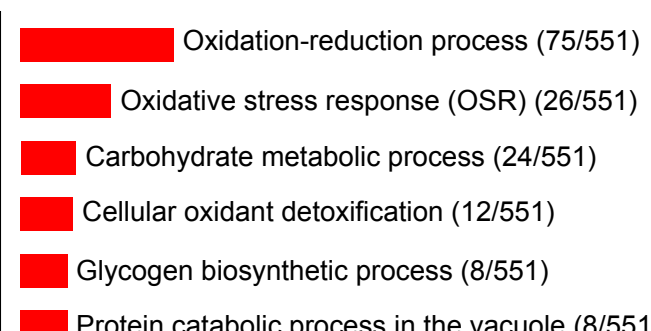

Cytoplasmic translation (46/138)

Translation (50/138)

Ribosome biogenesis (38/138)

RNA processing (33/138)

Ribosomal large subunit biogenesis (14/138)

$\begin{array}{llllll}0 & 10 & 20 & 30 & 40 & -\log _{10} P\end{array}$

\section{OSR}

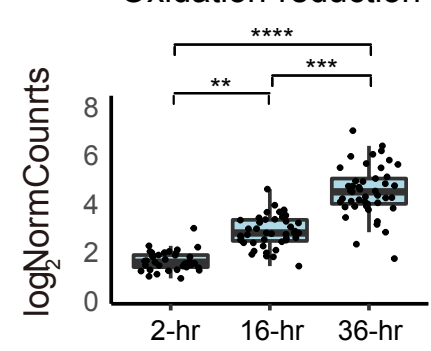

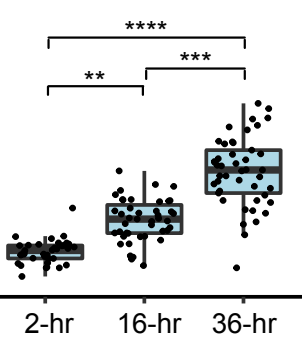

Mitochondrion(145 genes) Translation
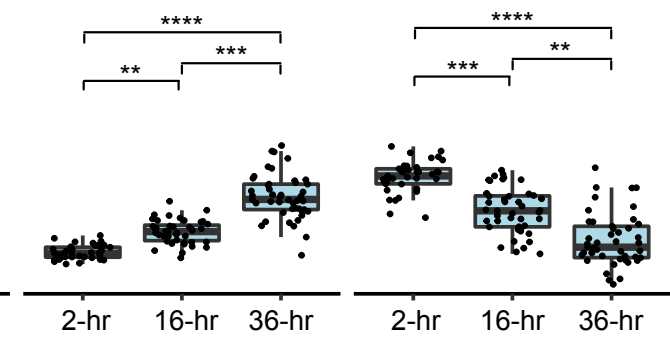

Ribosome biogeneiss

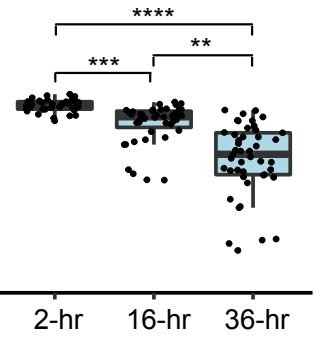


bioRxiv preprint doi: https://doi.org/10.1101/2020.09.04.282525; this version posted September 4, 2020. The copyright holder for this preprint (which was not certified by peer review) is the author/funder, who has granted bioRxiv a license to display the preprint in perpetuity. It is made available under aCC-BY-NC-ND 4.0 International license.

Figure.2, Zhang et al.

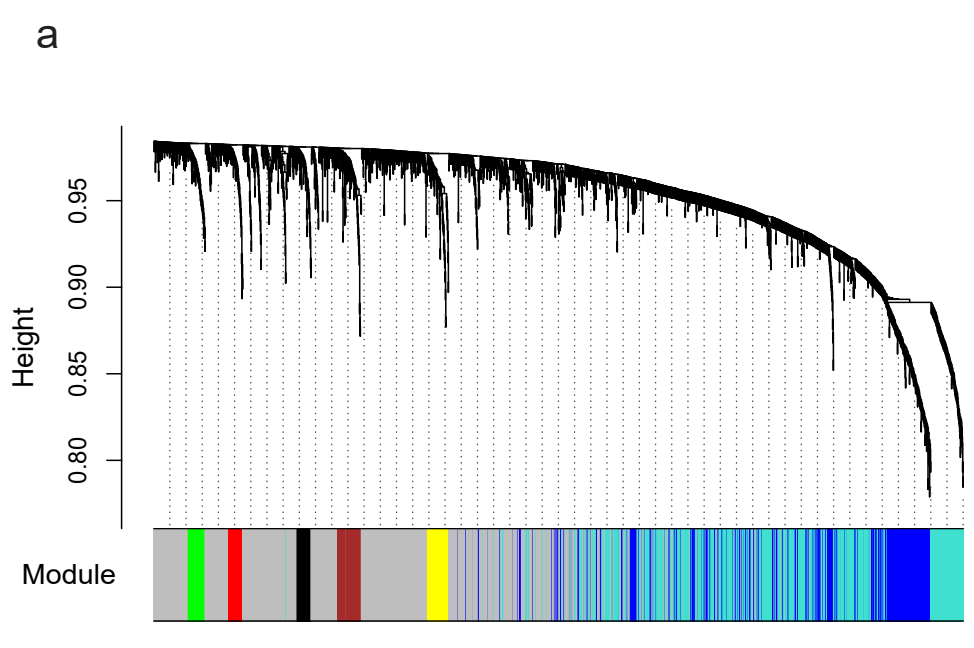

b

Module-trait relationships

C

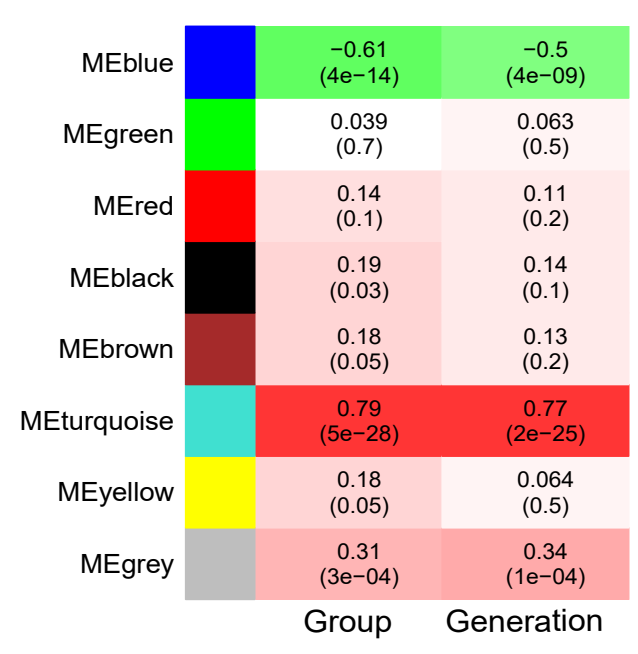

2-hr

16-hr

36-hr

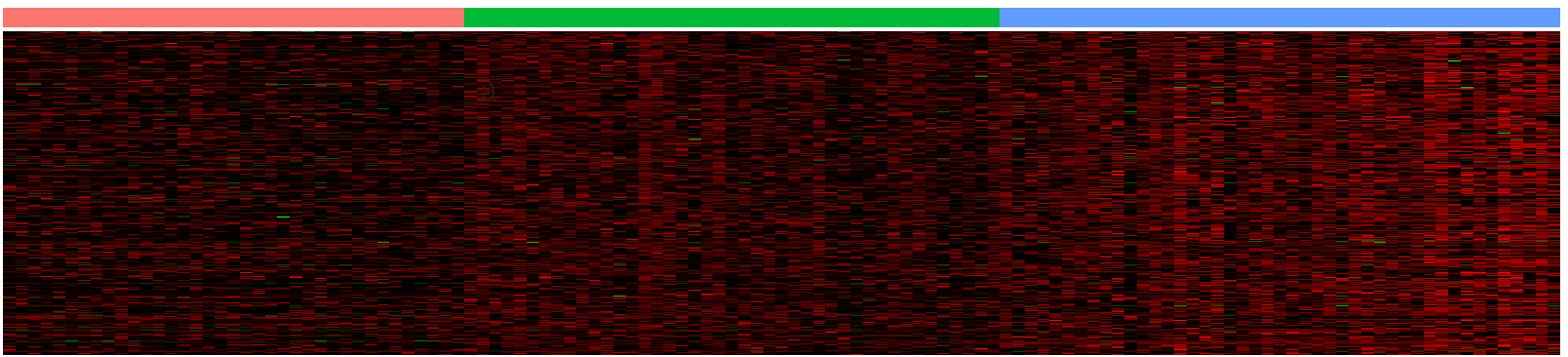

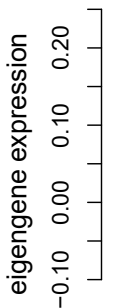

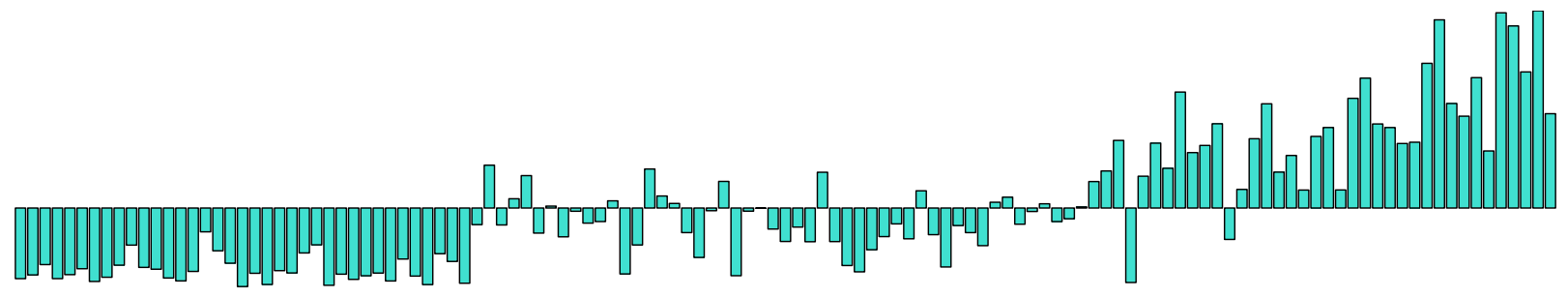

d

2-hr

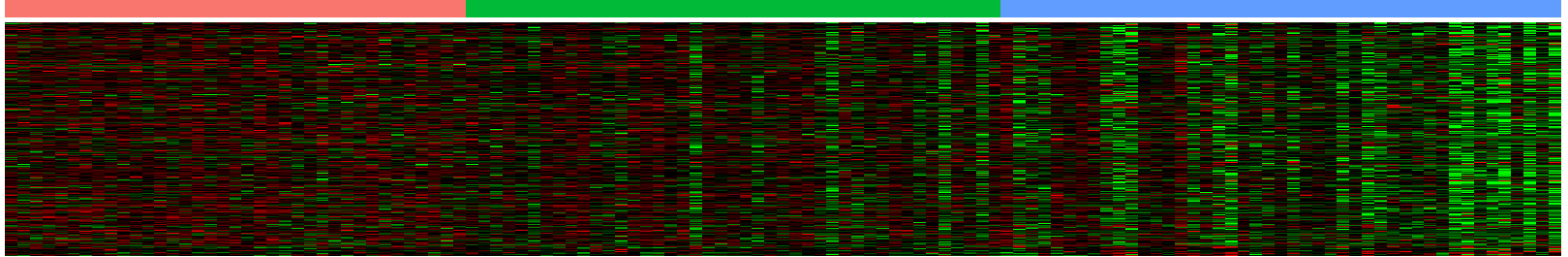

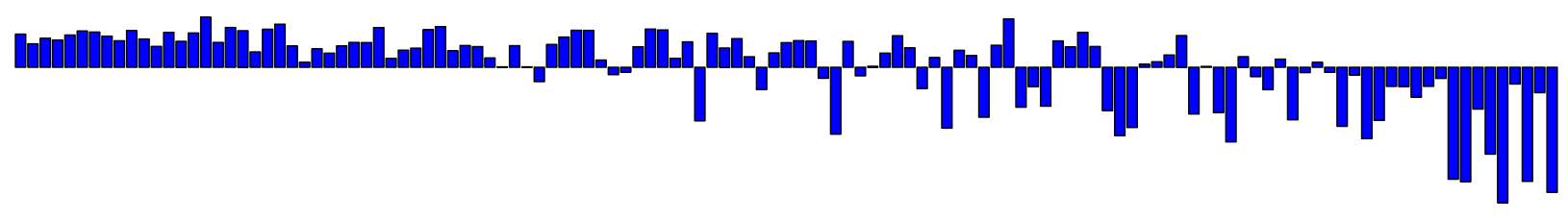


Figure.3, Zhang et afrpetuity. It is made available under aCC-BY-NC-ND 4.0 International license.

a

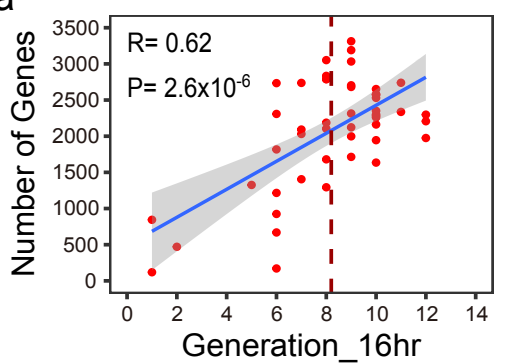

b

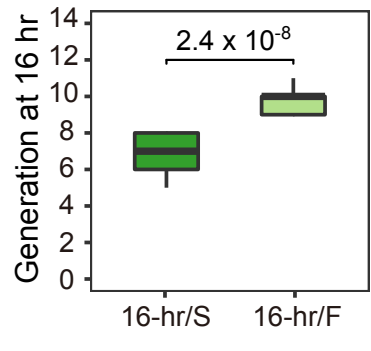

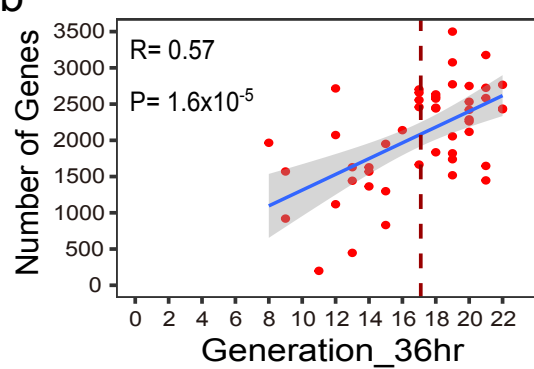

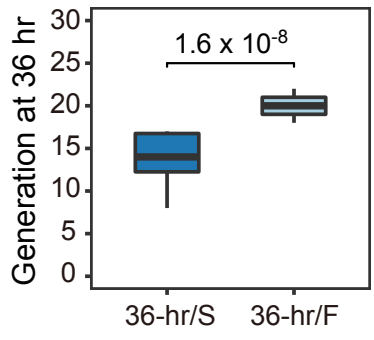

C

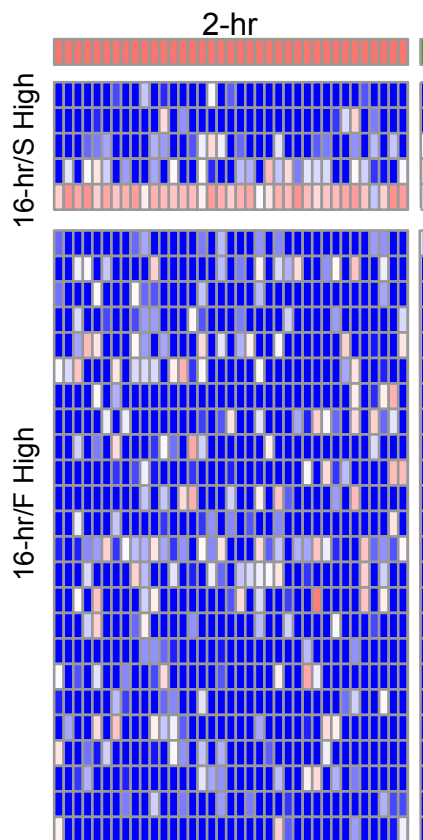

16-hr/S

16-hr/F

$36-\mathrm{hr} / \mathrm{S}$

36-hr/F

||

\section{H|

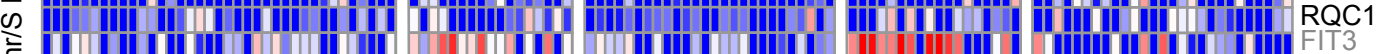

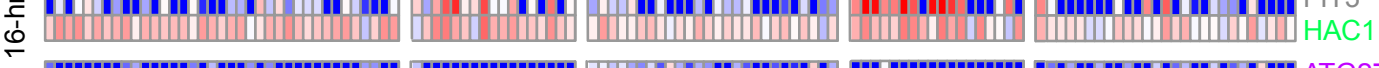

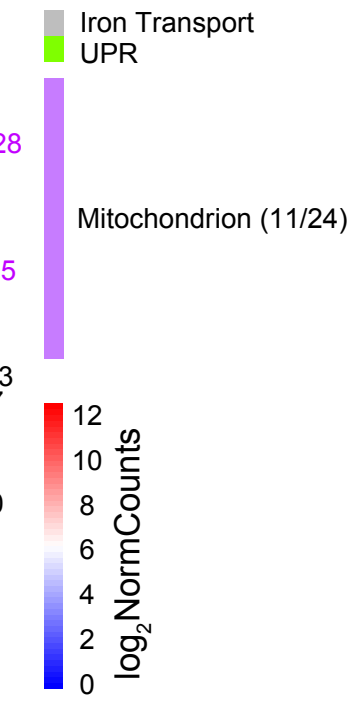

d

FIT3

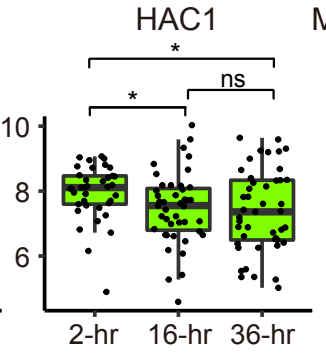

Mitochondrion (11 genes)
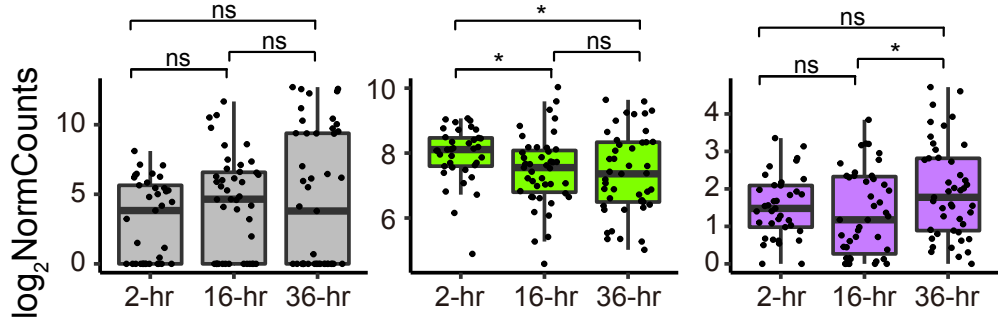

FIT3

HAC1

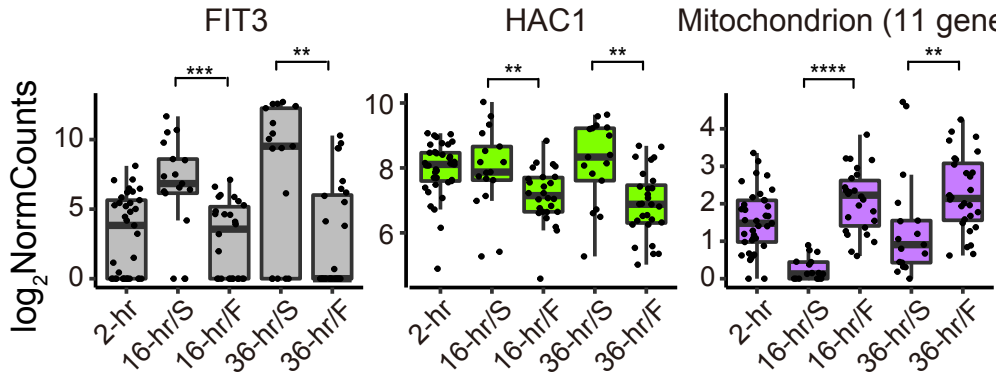

Mitochondrion (145 genes)

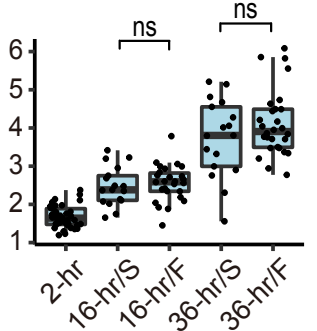

f

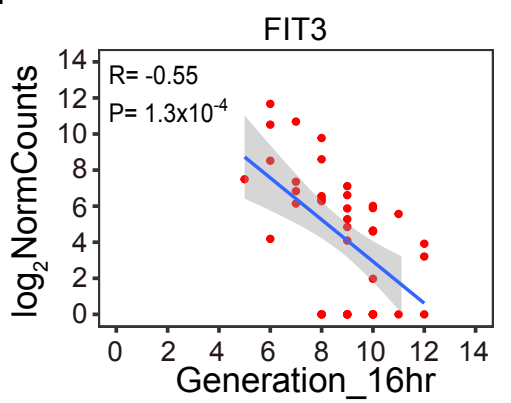

g

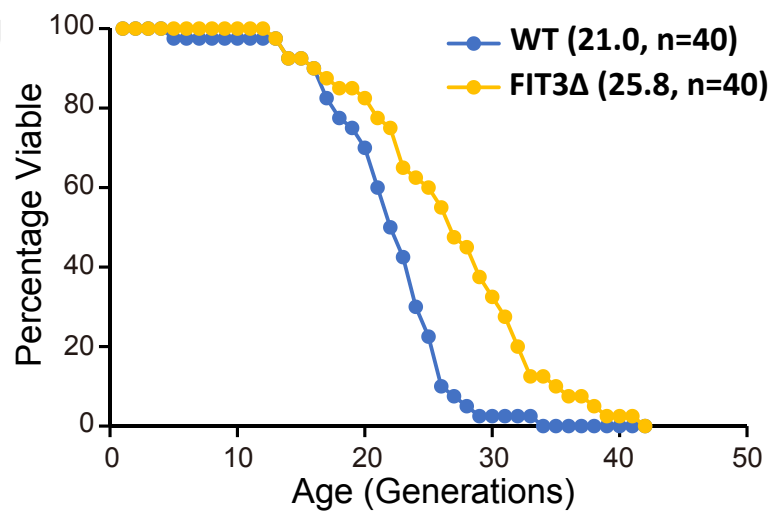


bioRxiv preprint doi: https://doi.org/10.1101/2020.09.04.282525; this version posted September 4, 2020. The copyright holder for this preprint (which was not certified by peer review) is the author/funder, who has granted bioRxiv a license to display the preprint in

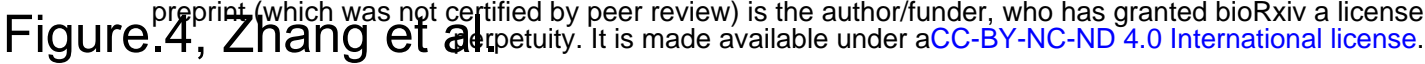

a

2-hr

$16-\mathrm{hr} / \mathrm{S}$

$16-\mathrm{hr} / \mathrm{F}$

$36-\mathrm{hr} / \mathrm{S}$

36-hr/F
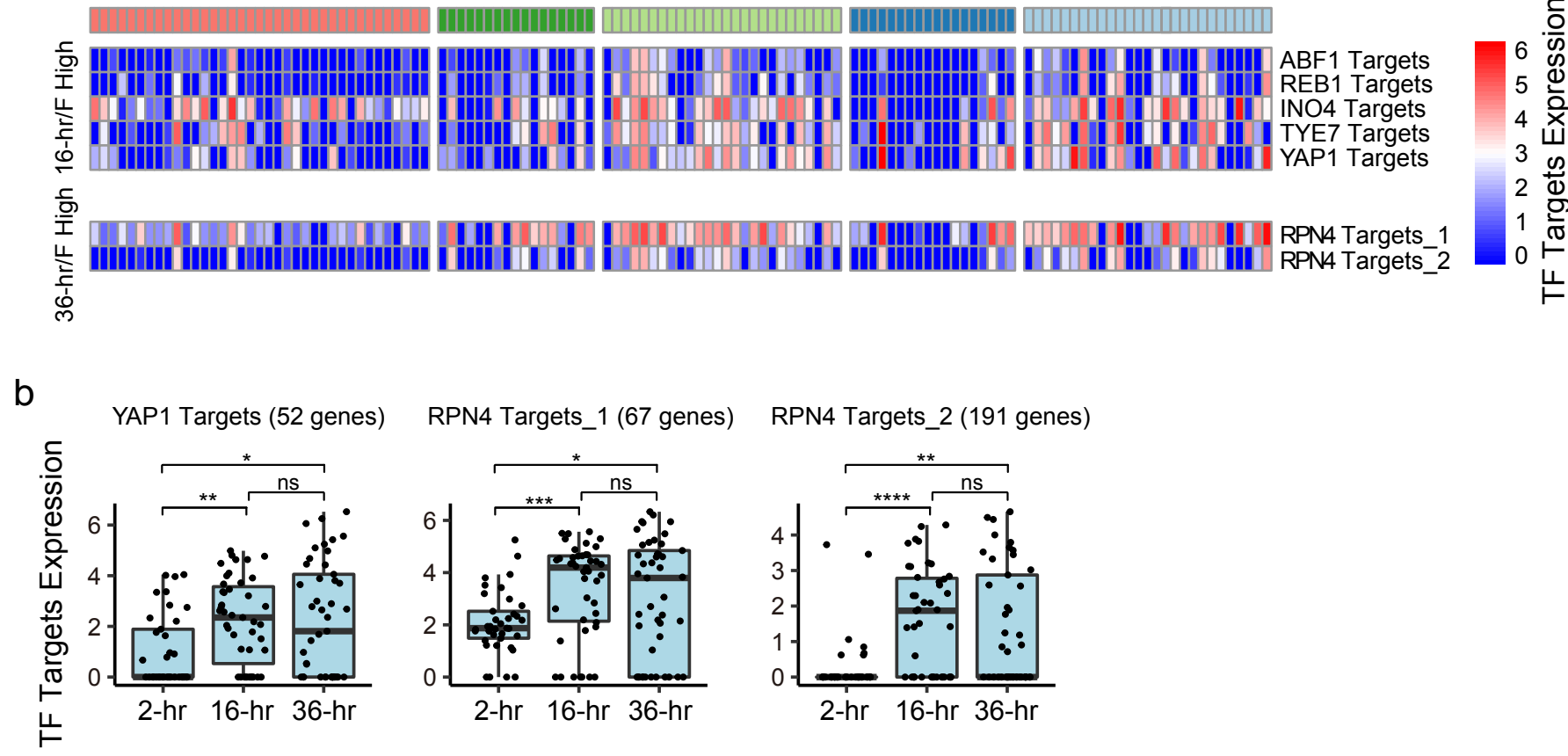

RPN4 Targets_1 (67 genes) RPN4 Targets_2 (191 genes)

C
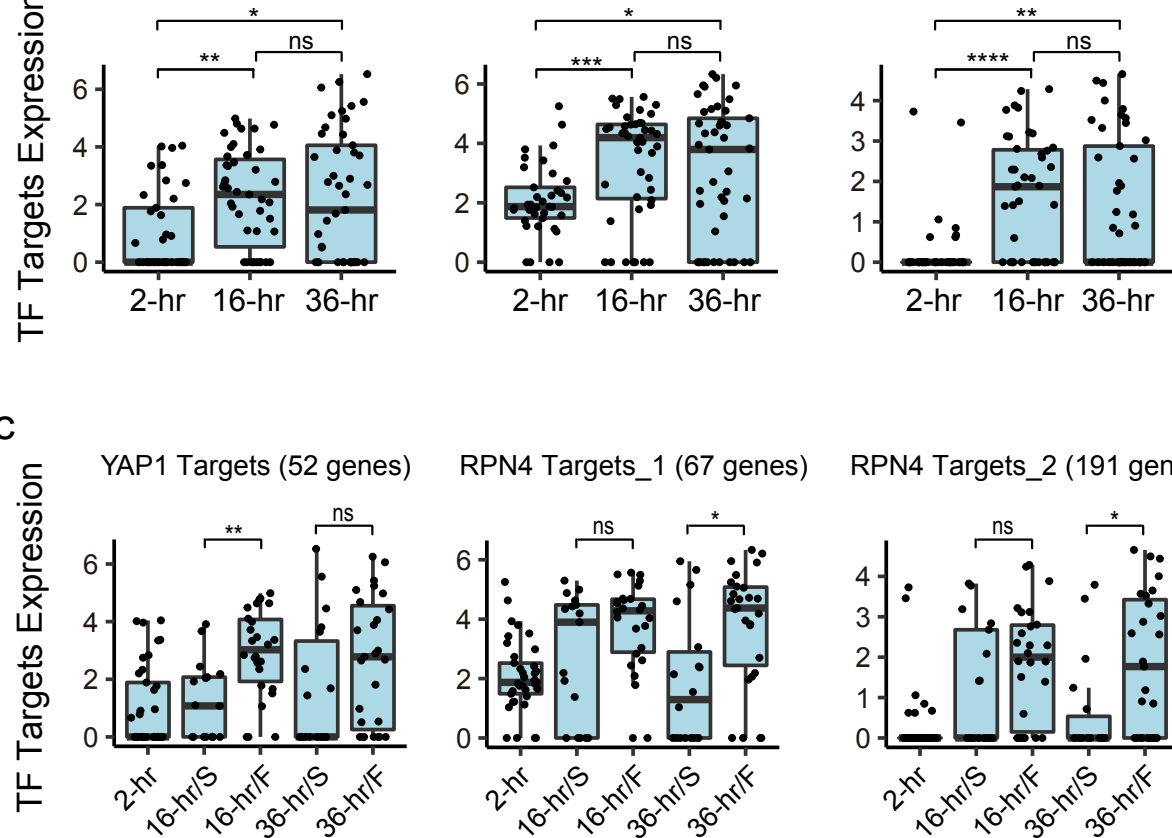

RPN4 Targets_1 (67 genes) RPN4 Targets_2 (191 genes)
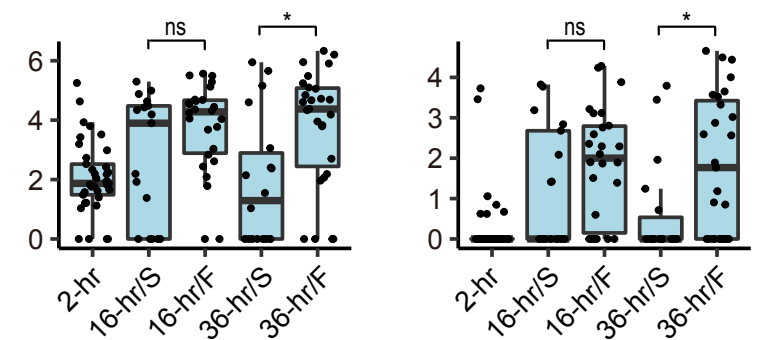